International Journal of Pure and Applied Mathematics

Volume 90 No. 4 2014, 501-513

ISSN: 1311-8080 (printed version); ISSN: 1314-3395 (on-line version)

url: http://www.ijpam.eu

doi: http://dx.doi.org/10.12732/ijpam.v90i4.11

ijpam.eu

\title{
REFINED ESTIMATORS OF MEASURES FOR MARGINAL HOMOGENEITY IN SQUARE CONTINGENCY TABLES
}

\author{
Kouji Tahata ${ }^{1 \S}$, Hiroyuki Tanaka ${ }^{2}$, Sadao Tomizawa ${ }^{3}$ \\ $1,2,3$ Department of Information Sciences \\ Faculty of Science and Technology \\ Tokyo University of Science \\ 2641 Yamazaki, Noda City, Chiba, 278-8510, JAPAN
}

\begin{abstract}
For square contingency tables, Tomizawa [6], Tomizawa and Makii [7] and Tomizawa, Miyamoto and Ashihara [8] considered the measures to represent the degree of departure from the marginal homogeneity model.

Using the first-order term in the Taylor series expansion, the estimated measures with the cell probabilities replaced by the corresponding sample proportions are approximately unbiased estimators of the corresponding measures when the sample size is large.

This paper proposes the refined approximate unbiased estimators of the measures which are obtained by using the second-order term in the Taylor series expansion.

The improved estimators approach to the true measures faster than the original estimators as the sample size becomes larger.

These are shown by the simulation studies.
\end{abstract}

AMS Subject Classification: $62 \mathrm{H} 17$

Key Words: estimation, marginal homogeneity, square contingency table, Taylor series expansion, unbiased estimators

\section{Introduction}

Consider an $R \times R$ square contingency table with same row and column clas-

Received: October 30, 2013

(c) 2014 Academic Publications, Ltd.

$\S$ Correspondence author url: www.acadpubl.eu 
sifications. Let $p_{i j}$ denote the probability that an observation will fall in the $i$ th row and $j$ th column of the table $(i=1,2, \ldots, R ; j=1,2, \ldots, R)$, and let $X_{1}$ and $X_{2}$ denote the row and column variables respectively. The marginal homogeneity $(\mathrm{MH})$ model is defined by

$$
p_{i \cdot}=p_{\cdot i} \quad(i=1,2, \ldots, R),
$$

where $p_{i}=\sum_{k=1}^{R} p_{i k}$ and $p_{\cdot i}=\sum_{k=1}^{R} p_{k i}$ (Stuart [4]). This model can be expressed as

$$
p_{i .}^{c}=p_{\cdot i}^{c} \quad(i=1,2, \ldots, R),
$$

where $p_{i \text {. }}^{c}=\left(p_{i \cdot}-p_{i i}\right) / \delta, p_{\cdot i}^{c}=\left(p_{\cdot i}-p_{i i}\right) / \delta$ and $\delta=\sum \sum_{i \neq j} p_{i j}$.

Let for $i=1,2, \ldots, R-1$

$$
G_{1(i)}=\sum_{s=1}^{i} \sum_{t=i+1}^{R} p_{s t} \quad \text { and } \quad G_{2(i)}=\sum_{s=i+1}^{R} \sum_{t=1}^{i} p_{s t}
$$

Then, the MH model may further be expressed as

$$
G_{1(i)}=G_{2(i)} \quad(i=1,2, \ldots, R-1)
$$

When the MH model does not hold for the given data, one may be interested in measuring the degree of departure from MH. Tomizawa [6] and Tomizawa and Makii [7] considered the measures $\Psi^{(\lambda)}$ and $\Phi^{(\lambda)}$ to represent the degree of departure from $\mathrm{MH}$ for the nominal data, which are expressed by using the power-divergence (Read and Cressie [3], p.15). Assuming that $\left\{p_{i}+p_{. i}\right\}$ are all positive, $\Psi^{(\lambda)}$ is defined by

$$
\Psi^{(\lambda)}=\frac{1}{2\left(2^{\lambda}-1\right)} \sum_{i=1}^{R}\left[p_{i \cdot}\left(\left(\frac{p_{i}}{\pi_{i}^{*}}\right)^{\lambda}-1\right)+p_{\cdot i}\left(\left(\frac{p_{\cdot i}}{\pi_{i}^{*}}\right)^{\lambda}-1\right)\right]
$$

for $\lambda>-1$, where $\pi_{i}^{*}=\left(p_{i}+p_{\cdot i}\right) / 2$ and $\Psi^{(0)}=\lim _{\lambda \rightarrow 0} \Psi^{(\lambda)}$. Also, $\Phi^{(\lambda)}$ is given as follows: assuming that $\left\{p_{i}^{c}+p_{. i}^{c}\right\}$ are all positive,

$$
\Phi^{(\lambda)}=\frac{1}{2\left(2^{\lambda}-1\right)} \sum_{i=1}^{R}\left[p_{i \cdot}^{c}\left(\left(\frac{p_{i \cdot}^{c}}{\pi_{i}^{c *}}\right)^{\lambda}-1\right)+p_{\cdot i}^{c}\left(\left(\frac{p_{\cdot i}^{c}}{\pi_{i}^{c *}}\right)^{\lambda}-1\right)\right]
$$

for $\lambda>-1$, where $\pi_{i}^{c *}=\left(p_{i}^{c}+p_{. i}^{c}\right) / 2$ and $\Phi^{(0)}=\lim _{\lambda \rightarrow 0} \Phi^{(\lambda)} \cdot \Psi^{(\lambda)}\left(\Phi^{(\lambda)}\right)$ is useful for seeing how far the unconditional (conditional) marginal distributions are distant from those with an $\mathrm{MH}$ structure. 
Tomizawa, Miyamoto and Ashihara [8] considered the measure $\Gamma^{(\lambda)}$ to represent the degree of departure from $\mathrm{MH}$ for the ordinal data. Assuming that $\left\{G_{1(i)}+G_{2(i)}\right\}$ are all positive, $\Gamma^{(\lambda)}$ is defined by

$$
\Gamma^{(\lambda)}=\frac{1}{2^{\lambda}-1} \sum_{i=1}^{R-1}\left[G_{1(i)}^{*}\left(\left(\frac{G_{1(i)}^{*}}{Q_{i}^{*}}\right)^{\lambda}-1\right)^{+} G_{2(i)}^{*}\left(\left(\frac{G_{2(i)}^{*}}{Q_{i}^{*}}\right)^{\lambda}-1\right)\right],
$$

for $\lambda>-1$, where $\Delta=\sum_{i=1}^{R-1}\left(G_{1(i)}+G_{2(i)}\right)$,

$$
G_{1(i)}^{*}=\frac{G_{1(i)}}{\Delta}, \quad G_{2(i)}^{*}=\frac{G_{2(i)}}{\Delta}, \quad Q_{i}^{*}=\frac{1}{2}\left(G_{1(i)}^{*}+G_{2(i)}^{*}\right),
$$

and $\Gamma^{(0)}=\lim _{\lambda \rightarrow 0} \Gamma^{(\lambda)}$.

The measures $\left(\Psi^{(\lambda)}, \Phi^{(\lambda)}\right.$ and $\left.\Gamma^{(\lambda)}\right)$ must lie between 0 and 1 and the degree of departure from $\mathrm{MH}$ increases as the value of measure increases. Also, these measures are useful for comparing between several tables.

Using the first-order term in the Taylor series expansion, the estimated measures with the cell probabilities replaced by the corresponding sample proportions are approximately unbiased estimators of the corresponding measures when the sample size is large. Using the second-order Taylor expansion, Tomizawa, Miyamoto and Ohba [9] improved the approximate unbiased estimators of measures of asymmetry for square contingency tables and Tahata, Tomisato and Tomizawa [5] also gave the improved approximate unbiased estimator of log-odds ratio. So we are now interested in, when the sample size $n$ is not so large, proposing the refined approximate unbiased estimators of $\Psi^{(\lambda)}, \Phi^{(\lambda)}$ and $\Gamma^{(\lambda)}$.

The purpose of this paper is to propose the improved approximate unbiased estimators of $\Psi^{(\lambda)}, \Phi^{(\lambda)}$ and $\Gamma^{(\lambda)}$. Section 2 gives these estimators. Section 3 shows that the proposed estimators work well in many cases by the simulation studies.

\section{Refined Approximate Unbiased Estimators}

Assume that the observed frequencies $\left\{n_{i j}\right\}$ have a multinomial distribution. Let $p$ be the $R^{2} \times 1$ vector

$$
p=\left(p_{11}, p_{12}, \ldots, p_{1 R}, p_{21}, p_{22}, \ldots, p_{2 R}, \ldots, p_{R 1}, p_{R 2}, \ldots, p_{R R}\right)^{t},
$$

where " $t$ " means transpose. Also let $\hat{p}_{i j}$ be sample proportion (i.e., $\hat{p}_{i j}=n_{i j} / n$ where $\left.n=\sum \sum n_{i j}\right)$ and let $\hat{p}$ be the $R^{2} \times 1$ vector in the similar way. We 
assume that $g$ has a nonzero differential at $p$, i.e., that $g$ has the following expansion as $\hat{p} \rightarrow p$ :

$$
g(\hat{p})=g(p)+\left[\frac{\partial g(p)}{\partial p^{t}}\right](\hat{p}-p)+o(\|\hat{p}-p\|),
$$

where $\left[\partial g(p) / \partial p^{t}\right]$ denotes $\left[\partial g(\hat{p}) / \partial \hat{p}^{t}\right]$ evaluated at $\hat{p}=p$. For the details, see e.g., Agresti ([1], p.589) and Bishop, Fienberg and Holland ([2], p.486). For large $n$, we can see from above equation that $g(\hat{p})$ is an approximate unbiased estimator of $g(p)$ because mean of $\hat{p}$ equals $p$. Similarly, the sample version of $\Psi^{(\lambda)}\left(\Phi^{(\lambda)}\right.$ and $\left.\Gamma^{(\lambda)}\right)$, i.e., $\hat{\Psi}^{(\lambda)}\left(\hat{\Phi}^{(\lambda)}\right.$ and $\left.\hat{\Gamma}^{(\lambda)}\right)$ is given by $\Psi^{(\lambda)}\left(\Phi^{(\lambda)}\right.$ and $\left.\Gamma^{(\lambda)}\right)$ with $p_{i j}$ replaced by $\hat{p}_{i j}$, is asymptotically unbiased estimator of $\Psi^{(\lambda)}\left(\Phi^{(\lambda)}\right.$ and $\left.\Gamma^{(\lambda)}\right)$ when the sample size $n$ is large.

Assuming that $g$ has a second differential at $p, g(\hat{p})$ has the following expansion as $\hat{p} \rightarrow p$ :

$$
\begin{aligned}
g(\hat{p})=g(p)+\left[\frac{\partial g(p)}{\partial p^{t}}\right](\hat{p}-p) & \\
& +\frac{1}{2}(\hat{p}-p)^{t}\left[\frac{\partial^{2} g(p)}{\partial p \partial p^{t}}\right](\hat{p}-p)+o\left(\|\hat{p}-p\|^{2}\right),
\end{aligned}
$$

where $\left[\partial^{2} g(p) / \partial p \partial p^{t}\right]$ denotes $\left[\partial^{2} g(\hat{p}) / \partial \hat{p} \partial \hat{p}^{t}\right]$ evaluated at $\hat{p}=p$. Therefore when the sample size $n$ is large, the mean of $g(\hat{p})$, i.e., $E(g(\hat{p}))$, is approximately equal to

$$
g(p)+\frac{1}{2 n} \operatorname{tr}\left(\left[\frac{\partial^{2} g(p)}{\partial p \partial p^{t}}\right]\left(D(p)-p p^{t}\right)\right),
$$

where $D(p)$ denotes the $R^{2} \times R^{2}$ diagonal matrix with the $i$ th element of $p$ as the $i$ th diagonal element, because $\operatorname{Var}(\hat{p})=\frac{1}{n}\left(D(p)-p p^{t}\right)$. Thus the mean of

$$
g(\hat{p})-\frac{1}{2 n} \operatorname{tr}\left(\left[\frac{\partial^{2} g(p)}{\partial p \partial p^{t}}\right]\left(D(p)-p p^{t}\right)\right)
$$

is approximately equal to $g(p)$, and it would approach to $g(p)$ faster than $g(\hat{p})$ as the sample size $n$ becomes larger. However, since the second term is unknown, the refined estimator of $g(p)$ is given as follows:

$$
g(\hat{p})-\frac{1}{2 n} \operatorname{tr}\left(\left[\frac{\partial^{2} g(\hat{p})}{\partial \hat{p} \partial \hat{p}^{t}}\right]\left(D(\hat{p})-\hat{p} \hat{p}^{t}\right)\right),
$$

where $\left[\partial^{2} g(\hat{p}) / \partial \hat{p} \partial \hat{p}^{t}\right]$ is given by $\left[\partial^{2} g(p) / \partial p \partial p^{t}\right]$ with $p_{i j}$ replaced by $\hat{p}_{i j}$ and $D(\hat{p})$ denotes $D(p)$ with $p_{i j}$ replaced by $\hat{p}_{i j}$. 
(1) We now propose the refined estimator of the true measure $\Psi^{(\lambda)}$ as follows:

$$
\hat{\Psi}^{*(\lambda)}=\hat{\Psi}^{(\lambda)}-\frac{1}{2 n} \operatorname{tr}\left(\left[\frac{\partial^{2} \hat{\Psi}^{(\lambda)}}{\partial \hat{p} \partial \hat{p}^{t}}\right]\left(D(\hat{p})-\hat{p} \hat{p}^{t}\right)\right),
$$

where $\left[\partial^{2} \hat{\Psi}^{(\lambda)} / \partial \hat{p} \partial \hat{p}^{t}\right]$ is given by $\left[\partial^{2} \Psi^{(\lambda)} / \partial p \partial p^{t}\right]$ with $p_{i j}$ replaced by $\hat{p}_{i j}$. The elements of $\left[\partial^{2} \Psi^{(\lambda)} / \partial p \partial p^{t}\right]$ are given as follows:

$$
\begin{aligned}
\frac{\partial^{2} \Psi^{(\lambda)}}{\partial p_{i j} \partial p_{k l}}=\frac{p_{2(i)}}{p_{i \cdot}+p_{\cdot i}} d_{(i, k)} K_{2(i)}^{(\lambda)}- & \frac{p_{1(i)}}{p_{i \cdot}+p_{\cdot i}} d_{(i, l)} K_{2(i)}^{(\lambda)} \\
& -\frac{p_{2(j)}}{p_{j \cdot}+p_{\cdot j}} d_{(j, k)} K_{1(j)}^{(\lambda)}+\frac{p_{1(j)}}{p_{j \cdot}+p_{\cdot j}} d_{(j, l)} K_{1(j)}^{(\lambda)},
\end{aligned}
$$

where $p_{1(i)}=p_{i \cdot} /\left(p_{i \cdot}+p_{\cdot i}\right), p_{2(i)}=p_{\cdot i} /\left(p_{i}+p_{\cdot i}\right)$ and

$$
\begin{gathered}
K_{1(j)}^{(\lambda)}=\frac{2^{\lambda} \lambda(\lambda+1)}{2\left(2^{\lambda}-1\right)}\left[p_{1(j)}\left(\left(p_{1(j)}\right)^{\lambda-1}+\left(p_{2(j)}\right)^{\lambda-1}\right)\right], \\
K_{2(i)}^{(\lambda)}=\frac{2^{\lambda} \lambda(\lambda+1)}{2\left(2^{\lambda}-1\right)}\left[p_{2(i)}\left(\left(p_{1(i)}\right)^{\lambda-1}+\left(p_{2(i)}\right)^{\lambda-1}\right)\right], \\
d_{(a, b)}= \begin{cases}1 & (a=b), \quad K_{1(j)}^{(0)}=\frac{1}{2(\log 2) p_{2(j)}}, \quad K_{2(i)}^{(0)}=\frac{1}{2(\log 2) p_{1(i)}} .\end{cases}
\end{gathered}
$$

(2) In the similar manner, we shall improve the estimated measure $\hat{\Phi}^{(\lambda)}$ as follows:

$$
\hat{\Phi}^{*(\lambda)}=\hat{\Phi}^{(\lambda)}-\frac{1}{2 n} \operatorname{tr}\left(\left[\frac{\partial^{2} \hat{\Phi}^{(\lambda)}}{\partial \hat{p} \partial \hat{p}^{t}}\right]\left(D(\hat{p})-\hat{p} \hat{p}^{t}\right)\right),
$$

where $\left[\partial^{2} \hat{\Phi}^{(\lambda)} / \partial \hat{p} \partial \hat{p}^{t}\right]$ is given by $\left[\partial^{2} \Phi^{(\lambda)} / \partial p \partial p^{t}\right]$ with $p_{i j}$ replaced by $\hat{p}_{i j}$. The elements of $\left[\partial^{2} \Phi^{(\lambda)} / \partial p \partial p^{t}\right]$ are given as follows:

$$
\begin{aligned}
\frac{\partial^{2} \Phi^{(\lambda)}}{\partial p_{i j} \partial p_{k l}}= & \frac{1}{\delta^{2}}\left[-\left(K_{i j}^{(\lambda)}+K_{k l}^{(\lambda)}\right)+\left(\frac{p_{2(i)}^{c}}{p_{i \cdot}^{c}+p_{\cdot i}^{c}} d_{(i, k)} L_{1(i)}^{(\lambda)}\right.\right. \\
& \left.\left.-\frac{p_{1(i)}^{c}}{p_{i \cdot}^{c}+p_{\cdot i}^{c}} d_{(i, l)} L_{1(i)}^{(\lambda)}-\frac{p_{2(j)}^{c}}{p_{j .}^{c}+p_{\cdot j}^{c}} d_{(j, k)} L_{2(j)}^{(\lambda)}+\frac{p_{1(j)}^{c}}{p_{j \cdot}^{c}+p_{\cdot j}^{c}} d_{(j, l)} L_{2(j)}^{(\lambda)}\right)\right],
\end{aligned}
$$

for $i \neq j$ and $k \neq l$, and $\partial^{2} \Phi^{(\lambda)} / \partial p_{i j} \partial p_{k l}=0$ for $i=j$ and/or $k=l$, where $p_{1(i)}^{c}=p_{i .}^{c} /\left(p_{i .}^{c}+p_{\cdot i}^{c}\right), p_{2(i)}^{c}=p_{. i}^{c} /\left(p_{i .}^{c}+p_{\cdot i}^{c}\right)$ and 


$$
\begin{gathered}
K_{i j}^{(\lambda)}=\frac{2^{\lambda-1}}{2^{\lambda}-1}\left[\left(p_{1(i)}^{c}\right)^{\lambda}+\left(p_{2(j)}^{c}\right)^{\lambda}+\lambda p_{2(i)}^{c}\left(\left(p_{1(i)}^{c}\right)^{\lambda}-\left(p_{2(i)}^{c}\right)^{\lambda}\right)\right. \\
\left.-\lambda p_{1(j)}^{c}\left(\left(p_{1(j)}^{c}\right)^{\lambda}-\left(p_{2(j)}^{c}\right)^{\lambda}\right)-\frac{2\left(\left(2^{\lambda}-1\right) \Phi^{(\lambda)}+1\right)}{2^{\lambda}}\right], \\
L_{1(i)}^{(\lambda)}=\frac{\lambda 2^{\lambda-1}}{2^{\lambda}-1}\left[\left(p_{1(i)}^{c}\right)^{\lambda-1}+\lambda p_{2(i)}^{c}\left(\left(p_{1(i)}^{c}\right)^{\lambda-1}+\left(p_{2(i)}^{c}\right)^{\lambda-1}\right)\right. \\
\left.-\left(\left(p_{1(i)}^{c}\right)^{\lambda}-\left(p_{2(i)}^{c}\right)^{\lambda}\right)\right], \\
L_{2(i)}^{(\lambda)}=\frac{\lambda 2^{\lambda-1}}{2^{\lambda}-1}\left[\left(p_{2(i)}^{c}\right)^{\lambda-1}+\lambda p_{1(i)}^{c}\left(\left(p_{1(i)}^{c}\right)^{\lambda-1}+\left(p_{2(i)}^{c}\right)^{\lambda-1}\right)\right. \\
\left.-\left(\left(p_{2(i)}^{c}\right)^{\lambda}-\left(p_{1(i)}^{c}\right)^{\lambda}\right)\right], \\
L_{1(i)}^{(0)}=\frac{1}{2(\log 2) p_{1(i)}^{c}}, L_{2(i)}^{(0)}=\frac{1}{2(\log 2) p_{2(i)}^{c}} . \\
K_{i j}^{(0)}=\frac{\log \left(p_{1(i)}^{c} p_{2(j)}^{c}\right)}{2 \log 2}+\left(1-\Phi^{(0)}\right),
\end{gathered}
$$

(3) Finally, we shall improve the estimated measure $\hat{\Gamma}^{(\lambda)}$ as follows:

$$
\hat{\Gamma}^{*(\lambda)}=\hat{\Gamma}^{(\lambda)}-\frac{1}{2 n} \operatorname{tr}\left(\left[\frac{\partial^{2} \hat{\Gamma}^{(\lambda)}}{\partial \hat{p} \partial \hat{p}^{t}}\right]\left(D(\hat{p})-\hat{p} \hat{p}^{t}\right)\right),
$$

where $\left[\partial^{2} \hat{\Gamma}^{(\lambda)} / \partial \hat{p} \partial \hat{p}^{t}\right]$ is given by $\left[\partial^{2} \Gamma^{(\lambda)} / \partial p \partial p^{t}\right]$ with $p_{i j}$ replaced by $\hat{p}_{i j}$. The elements of $\left[\partial^{2} \Gamma^{(\lambda)} / \partial p \partial p^{t}\right]$ are given as follows:

$$
\begin{aligned}
\frac{\partial^{2} \Gamma^{(\lambda)}}{\partial p_{i j} \partial p_{k l}}=\frac{1}{\Delta}\left[I _ { ( i < j ) } \left(L_{1(i j ; k l)}^{(\lambda)}-|k-l|\right.\right. & \left.W_{1(i j)}^{(\lambda)}\right) \\
& \left.+I_{(i>j)}\left(L_{2(i j ; k l)}^{(\lambda)}-|k-l| W_{2(i j)}^{(\lambda)}\right)\right]
\end{aligned}
$$

where $I_{(\cdot)}=1$ if true, 0 if not, and

$$
W_{1(i j)}^{(\lambda)}=\frac{2^{\lambda}}{\Delta\left(2^{\lambda}-1\right)}\left[\sum_{s=i}^{j-1}\left\{\left(G_{1(s)}^{c}\right)^{\lambda}+\lambda\left(\left(G_{1(s)}^{c}\right)^{\lambda}-\left(G_{2(s)}^{c}\right)^{\lambda}\right) G_{2(s)}^{c}\right\}\right.
$$


Table 1: (a) The artificial probabilities $\left\{p_{i j}\right\}$, (b) the values of $\Psi^{(\lambda)}$, $\Phi^{(\lambda)}$ and $\Gamma^{(\lambda)}$, and (c) the each sample mean of the values of estimated measures obtained by generating 10000 times simulations, with each sample size $n$, for Table 1(a)

(a)

\begin{tabular}{ccccc}
\hline & $(1)$ & $(2)$ & $(3)$ & $(4)$ \\
\hline$(1)$ & 0.050 & 0.043 & 0.036 & 0.037 \\
$(2)$ & 0.105 & 0.050 & 0.122 & 0.072 \\
$(3)$ & 0.042 & 0.024 & 0.050 & 0.030 \\
$(4)$ & 0.118 & 0.051 & 0.120 & 0.050 \\
\hline
\end{tabular}

\begin{tabular}{cccc} 
(b) & & & \\
\hline$\lambda$ & $\Psi^{(\lambda)}$ & $\Phi^{(\lambda)}$ & $\Gamma^{(\lambda)}$ \\
\hline
\end{tabular}

$\begin{array}{llll}1.0 & 0.1110 & 0.1737 & 0.0861\end{array}$

\begin{tabular}{llll}
1.5 & 0.1136 & 0.1775 & 0.0880 \\
\hline
\end{tabular}

(c)

\begin{tabular}{cccccccc}
\hline$n$ & $\lambda$ & $\hat{\Psi}^{(\lambda)}$ & $\hat{\Psi}^{*(\lambda)}$ & $\hat{\Phi}^{(\lambda)}$ & $\hat{\Phi}^{*(\lambda)}$ & $\hat{\Gamma}^{(\lambda)}$ & $\hat{\Gamma}^{*(\lambda)}$ \\
\hline 50 & 1.0 & 0.1399 & 0.1124 & 0.2148 & 0.1754 & 0.1270 & 0.0869 \\
& 1.5 & 0.1418 & 0.1140 & 0.2188 & 0.1795 & 0.1301 & 0.0894 \\
100 & 1.0 & 0.1260 & 0.1120 & 0.1937 & 0.1735 & 0.1070 & 0.0865 \\
& 1.5 & 0.1275 & 0.1134 & 0.1975 & 0.1773 & 0.1086 & 0.0878 \\
200 & 1.0 & 0.1181 & 0.1110 & 0.1839 & 0.1737 & 0.0963 & 0.0860 \\
& 1.5 & 0.1210 & 0.1139 & 0.1877 & 0.1775 & 0.0988 & 0.0884 \\
400 & 1.0 & 0.1147 & 0.1111 & 0.1791 & 0.1739 & 0.0913 & 0.0861 \\
& 1.5 & 0.1171 & 0.1135 & 0.1828 & 0.1777 & 0.0937 & 0.0885 \\
800 & 1.0 & 0.1129 & 0.1111 & 0.1765 & 0.1739 & 0.0889 & 0.0863 \\
& 1.5 & 0.1154 & 0.1136 & 0.1803 & 0.1777 & 0.0905 & 0.0878 \\
\hline
\end{tabular}

$$
\left.-(j-i) \frac{\left(2^{\lambda}-1\right) \Gamma^{(\lambda)}+1}{2^{\lambda}}\right],
$$

$$
\begin{array}{r}
W_{2(i j)}^{(\lambda)}=\frac{2^{\lambda}}{\Delta\left(2^{\lambda}-1\right)}\left[\sum_{s=j}^{i-1}\left\{\left(G_{2(s)}^{c}\right)^{\lambda}+\lambda\left(\left(G_{2(s)}^{c}\right)^{\lambda}-\left(G_{1(s)}^{c}\right)^{\lambda}\right) G_{1(s)}^{c}\right\}\right. \\
\left.-(i-j) \frac{\left(2^{\lambda}-1\right) \Gamma^{(\lambda)}+1}{2^{\lambda}}\right],
\end{array}
$$


Table 2: (a) The artificial probabilities $\left\{p_{i j}\right\}$, (b) the values of $\Psi^{(\lambda)}$, $\Phi^{(\lambda)}$ and $\Gamma^{(\lambda)}$, and (c) the each sample mean of the values of estimated measures obtained by generating 10000 times simulations, with each sample size $n$, for Table $2(\mathrm{a})$

\section{(a)}

\begin{tabular}{ccccc}
\hline & $(1)$ & $(2)$ & $(3)$ & $(4)$ \\
\hline$(1)$ & 0.003 & 0.037 & 0.229 & 0.145 \\
$(2)$ & 0.023 & 0.003 & 0.141 & 0.245 \\
$(3)$ & 0.021 & 0.019 & 0.003 & 0.043 \\
$(4)$ & 0.025 & 0.031 & 0.029 & 0.003 \\
\hline
\end{tabular}

(b)

\begin{tabular}{cccc}
\hline$\lambda$ & $\Psi^{(\lambda)}$ & $\Phi^{(\lambda)}$ & $\Gamma^{(\lambda)}$ \\
\hline 1.0 & 0.4415 & 0.4523 & 0.5353 \\
1.5 & 0.4483 & 0.4591 & 0.5423 \\
\hline
\end{tabular}

(c)

\begin{tabular}{cccccccc}
\hline$n$ & $\lambda$ & $\hat{\Psi}^{(\lambda)}$ & $\hat{\Psi}^{*(\lambda)}$ & $\hat{\Phi}^{(\lambda)}$ & $\hat{\Phi}^{*(\lambda)}$ & $\hat{\Gamma}^{(\lambda)}$ & $\hat{\Gamma}^{*(\lambda)}$ \\
\hline 50 & 1.0 & 0.4649 & 0.4438 & 0.4759 & 0.4547 & 0.5494 & 0.5347 \\
& 1.5 & 0.4691 & 0.4488 & 0.4821 & 0.4617 & 0.5561 & 0.5422 \\
100 & 1.0 & 0.4527 & 0.4419 & 0.4626 & 0.4517 & 0.5450 & 0.5376 \\
& 1.5 & 0.4577 & 0.4473 & 0.4689 & 0.4585 & 0.5498 & 0.5427 \\
200 & 1.0 & 0.4479 & 0.4424 & 0.4580 & 0.4525 & 0.5392 & 0.5355 \\
& 1.5 & 0.4524 & 0.4472 & 0.4641 & 0.4588 & 0.5459 & 0.5424 \\
400 & 1.0 & 0.4441 & 0.4414 & 0.4551 & 0.4524 & 0.5371 & 0.5353 \\
& 1.5 & 0.4504 & 0.4478 & 0.4612 & 0.4585 & 0.5446 & 0.5428 \\
800 & 1.0 & 0.4429 & 0.4415 & 0.4535 & 0.4522 & 0.5361 & 0.5351 \\
& 1.5 & 0.4499 & 0.4486 & 0.4602 & 0.4588 & 0.5428 & 0.5419 \\
\hline
\end{tabular}

$$
\begin{aligned}
L_{1(i j ; k l)}^{(\lambda)} & =\frac{2^{\lambda}}{2^{\lambda}-1} \sum_{s=i}^{j-1}\left[\frac { d _ { k l ( s ) } G _ { 2 ( s ) } ^ { c } - d _ { l k ( s ) } G _ { 1 ( s ) } ^ { c } } { G _ { 1 ( s ) } + G _ { 2 ( s ) } } \lambda \left(\left(G_{1(s)}^{c}\right)^{\lambda-1}\right.\right. \\
+ & \left.\left.\lambda G_{2(s)}^{c}\left(\left(G_{1(s)}^{c}\right)^{\lambda-1}+\left(G_{2(s)}^{c}\right)^{\lambda-1}\right)-\left(\left(G_{1(s)}^{c}\right)^{\lambda}-\left(G_{2(s)}^{c}\right)^{\lambda}\right)\right)\right] \\
& -(j-i)\left(I_{(k<l)} W_{1(k l)}^{(\lambda)}+I_{(k>l)} W_{2(k l)}^{(\lambda)}\right),
\end{aligned}
$$

$$
\begin{aligned}
L_{2(i j ; k l)}^{(\lambda)} & =\frac{2^{\lambda}}{2^{\lambda}-1} \sum_{s=j}^{i-1}\left[\frac { d _ { k l ( s ) } G _ { 2 ( s ) } ^ { c } - d _ { l k ( s ) } G _ { 1 ( s ) } ^ { c } } { G _ { 1 ( s ) } + G _ { 2 ( s ) } } \lambda \left(-\left(G_{2(s)}^{c}\right)^{\lambda-1}\right.\right. \\
& \left.\left.-\lambda G_{1(s)}^{c}\left(\left(G_{1(s)}^{c}\right)^{\lambda-1}+\left(G_{2(s)}^{c}\right)^{\lambda-1}\right)+\left(\left(G_{2(s)}^{c}\right)^{\lambda}-\left(G_{1(s)}^{c}\right)^{\lambda}\right)\right)\right]
\end{aligned}
$$


Table 3: (a) The artificial probabilities $\left\{p_{i j}\right\}$, (b) the values of $\Psi^{(\lambda)}$, $\Phi^{(\lambda)}$ and $\Gamma^{(\lambda)}$, and (c) the each sample mean of the values of estimated measures obtained by generating 10000 times simulations, with each sample size $n$, for Table 3(a)

\begin{tabular}{ccccc} 
(a) & & & & \\
\hline & $(1)$ & $(2)$ & $(3)$ & $(4)$ \\
\hline$(1)$ & 0.002 & 0.009 & 0.226 & 0.219 \\
$(2)$ & 0.008 & 0.002 & 0.251 & 0.224 \\
$(3)$ & 0.007 & 0.008 & 0.002 & 0.022 \\
$(4)$ & 0.005 & 0.006 & 0.007 & 0.002 \\
\hline
\end{tabular}

\begin{tabular}{cccc} 
(b) & & & \\
\hline$\lambda$ & $\Psi^{(\lambda)}$ & $\Phi^{(\lambda)}$ & $\Gamma^{(\lambda)}$ \\
\hline 1.0 & 0.7999 & 0.8129 & 0.8702 \\
1.5 & 0.8047 & 0.8174 & 0.8737 \\
\hline
\end{tabular}

(c)

\begin{tabular}{cccccccc}
\hline$n$ & $\lambda$ & $\hat{\Psi}^{(\lambda)}$ & $\hat{\Psi}^{*(\lambda)}$ & $\hat{\Phi}^{(\lambda)}$ & $\hat{\Phi}^{*(\lambda)}$ & $\hat{\Gamma}^{(\lambda)}$ & $\hat{\Gamma}^{*(\lambda)}$ \\
\hline 50 & 1.0 & 0.8056 & 0.7980 & 0.8180 & 0.8108 & 0.8747 & 0.8709 \\
& 1.5 & 0.8110 & 0.8042 & 0.8232 & 0.8167 & 0.8762 & 0.8728 \\
100 & 1.0 & 0.8047 & 0.8009 & 0.8164 & 0.8128 & 0.8720 & 0.8700 \\
& 1.5 & 0.8082 & 0.8047 & 0.8181 & 0.8174 & 0.8763 & 0.8747 \\
200 & 1.0 & 0.8020 & 0.8001 & 0.8152 & 0.8134 & 0.8718 & 0.8708 \\
& 1.5 & 0.8065 & 0.8048 & 0.8189 & 0.8172 & 0.8743 & 0.8735 \\
400 & 1.0 & 0.8011 & 0.8001 & 0.8139 & 0.8130 & 0.8708 & 0.8703 \\
& 1.5 & 0.8054 & 0.8045 & 0.8179 & 0.8171 & 0.8741 & 0.8737 \\
800 & 1.0 & 0.8002 & 0.7997 & 0.8132 & 0.8128 & 0.8707 & 0.8704 \\
& 1.5 & 0.8054 & 0.8050 & 0.8180 & 0.8176 & 0.8742 & 0.8740 \\
\hline
\end{tabular}

$$
-(i-j)\left(I_{(k<l)} W_{1(k l)}^{(\lambda)}+I_{(k>l)} W_{2(k l)}^{(\lambda)}\right),
$$

with $G_{1(i)}^{c}=G_{1(i)} /\left(G_{1(i)}+G_{2(i)}\right), G_{2(i)}^{c}=G_{2(i)} /\left(G_{1(i)}+G_{2(i)}\right)$,

$$
d_{k l(s)}= \begin{cases}1 & (k \leq s, l \geq s+1) \\ 0 & \text { (otherwise) }\end{cases}
$$

and

$$
\begin{aligned}
& W_{1(i j)}^{(0)}=\frac{1}{\Delta \log 2}\left[\sum_{s=i}^{j-1} \log G_{1(s)}^{c}-(j-i)\left(\Gamma^{(0)}-1\right) \log 2\right], \\
& W_{2(i j)}^{(0)}=\frac{1}{\Delta \log 2}\left[\sum_{s=j}^{i-1} \log G_{2(s)}^{c}-(i-j)\left(\Gamma^{(0)}-1\right) \log 2\right\rfloor,
\end{aligned}
$$


Table 4: (a) The artificial probabilities $\left\{p_{i j}\right\}$, (b) the values of $\Psi^{(\lambda)}$, $\Phi^{(\lambda)}$ and $\Gamma^{(\lambda)}$, and (c) the each sample mean of the values of estimated measures obtained by generating 10000 times simulations, with each sample size $n$, for Table $4(\mathrm{a})$

\section{(a)}

\begin{tabular}{ccccc}
\hline & $(1)$ & $(2)$ & $(3)$ & $(4)$ \\
\hline$(1)$ & 0.100 & 0.037 & 0.099 & 0.052 \\
$(2)$ & 0.062 & 0.100 & 0.086 & 0.096 \\
$(3)$ & 0.013 & 0.018 & 0.100 & 0.051 \\
$(4)$ & 0.018 & 0.020 & 0.048 & 0.100 \\
\hline
\end{tabular}

\begin{tabular}{cccc} 
(b) & & & \\
\hline$\lambda$ & $\Psi^{(\lambda)}$ & $\Phi^{(\lambda)}$ & $\Gamma^{(\lambda)}$ \\
\hline
\end{tabular}

$\begin{array}{llll}1.0 & 0.0722 & 0.1990 & 0.2586\end{array}$

$\begin{array}{llll}1.5 & 0.0739 & 0.2031 & 0.2632\end{array}$

(c)

\begin{tabular}{cccccccc}
\hline$n$ & $\lambda$ & $\hat{\Psi}^{(\lambda)}$ & $\hat{\Psi}^{*(\lambda)}$ & $\hat{\Phi}^{(\lambda)}$ & $\hat{\Phi}^{*(\lambda)}$ & $\hat{\Gamma}^{(\lambda)}$ & $\hat{\Gamma}^{*(\lambda)}$ \\
\hline 50 & 1.0 & 0.0953 & 0.0735 & 0.2547 & 0.2043 & 0.3080 & 0.2642 \\
& 1.5 & 0.0974 & 0.0754 & 0.2602 & 0.2100 & 0.3134 & 0.2699 \\
\multirow{2}{*}{100} & 1.0 & 0.0833 & 0.0723 & 0.2256 & 0.1995 & 0.2812 & 0.2586 \\
& 1.5 & 0.0858 & 0.0746 & 0.2306 & 0.2046 & 0.2867 & 0.2644 \\
200 & 1.0 & 0.0778 & 0.0722 & 0.2123 & 0.1991 & 0.2698 & 0.2584 \\
& 1.5 & 0.0801 & 0.0745 & 0.2182 & 0.2050 & 0.2763 & 0.2650 \\
400 & 1.0 & 0.0753 & 0.0725 & 0.2063 & 0.1997 & 0.2649 & 0.2592 \\
& 1.5 & 0.0764 & 0.0736 & 0.2089 & 0.2022 & 0.2679 & 0.2622 \\
800 & 1.0 & 0.0738 & 0.0724 & 0.2028 & 0.1995 & 0.2624 & 0.2595 \\
& 1.5 & 0.0752 & 0.0738 & 0.2064 & 0.2031 & 0.2658 & 0.2629 \\
\hline
\end{tabular}

$$
\begin{aligned}
L_{1(i j ; k l)}^{(0)}=\frac{1}{\log 2}\left[\sum_{s=i}^{j-1} \frac{d_{k l(s)} G_{2(s)}^{c}-d_{l k(s)} G_{1(s)}^{c}}{G_{1(s)}^{c}\left(G_{1(s)}+G_{2(s)}\right)}\right] \\
-(j-i)\left(I_{(k<l)} W_{1(k l)}^{(0)}+I_{(k>l)} W_{2(k l)}^{(0)}\right),
\end{aligned}
$$$$
L_{2(i j ; k l)}^{(0)}=\frac{-1}{\log 2}\left[\sum_{s=j}^{i-1} \frac{d_{k l(s)} G_{2(s)}^{c}-d_{l k(s)} G_{1(s)}^{c}}{G_{2(s)}^{c}\left(G_{1(s)}+G_{2(s)}\right)}\right]
$$$$
-(i-j)\left(I_{(k<l)} W_{1(k l)}^{(0)}+I_{(k>l)} W_{2(k l)}^{(0)}\right) .
$$ 
Table 5: (a) The artificial probabilities $\left\{p_{i j}\right\}$, (b) the values of $\Psi^{(\lambda)}$, $\Phi^{(\lambda)}$ and $\Gamma^{(\lambda)}$, and (c) the each sample mean of the values of estimated measures obtained by generating 10000 times simulations, with each sample size $n$, for Table $5(\mathrm{a})$

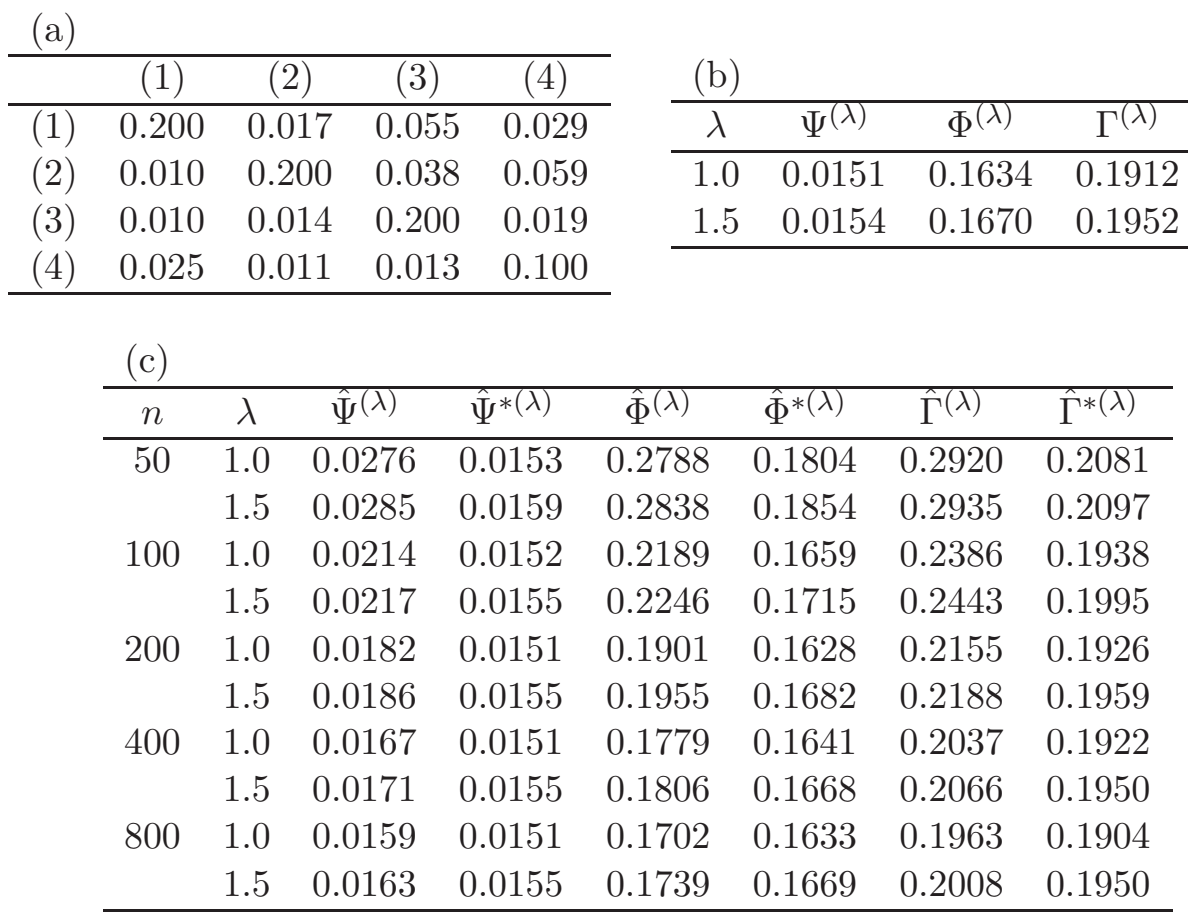

\section{Simulation Studies}

By the simulation studies, we calculate the values of estimated measures $\hat{\Psi}^{(\lambda)}$, $\hat{\Psi}^{*(\lambda)}, \hat{\Phi}^{(\lambda)}, \hat{\Phi}^{*(\lambda)}, \hat{\Gamma}^{(\lambda)}$ and $\hat{\Gamma}^{*(\lambda)}$ from the observed frequencies of sample size $n=50,100,200,400$ and 800, which are obtained from the true probability distribution. We shall compare the each sample mean of the values of $\hat{\Psi}^{(\lambda)}$ and $\hat{\Psi}^{*(\lambda)}$ obtained by 10000 times simulations, for each sample size. Similarly, the sample mean of the value of $\hat{\Phi}^{(\lambda)}\left(\hat{\Gamma}^{(\lambda)}\right)$ is compared to that of $\hat{\Phi}^{*(\lambda)}\left(\hat{\Gamma}^{*(\lambda)}\right)$. The results of simulations are given in Tables 1 to 5 .

Tables 1(a), 2(a) and 3(a) have a characteristic that the sum of the probabilities of main-diagonal cells is very small. Also the true values of measures for Tables 1(a), 2(a) and 3(a) are small, medium and large, respectively. The sums 
of the probabilities of main-diagonal cells for Tables 4(a) and 5(a) are greater than those for Tables 1(a), 2(a) and 3(a), and the true value of measures for Tables 4(a) and 5(a) are small.

We can see from Tables $1(\mathrm{c})$ to $5(\mathrm{c})$ that the refined estimator $\hat{\Psi}^{*(\lambda)}$ approaches to the true value $\Psi^{(\lambda)}$ faster than the former estimator $\hat{\Psi}^{(\lambda)}$ when $\lambda \geq 1$. Also we can obtain the similar results for the improved estimators $\hat{\Phi}^{*(\lambda)}$ and $\hat{\Gamma}^{*(\lambda)}$.

\section{Concluding Remarks}

This paper has proposed the refined approximate unbiased estimators $\hat{\Psi}^{*(\lambda)}$, $\hat{\Phi}^{*(\lambda)}$ and $\hat{\Gamma}^{*(\lambda)}$ of the true measures $\Psi^{(\lambda)}, \Phi^{(\lambda)}$ and $\Gamma^{(\lambda)}$, respectively.

From the simulation studies, we conclude that the improved estimator $\hat{\Psi}^{*(\lambda)}$ $\left(\hat{\Phi}^{*(\lambda)}\right.$ and $\left.\hat{\Gamma}^{*(\lambda)}\right)$ tends to approach to the true value $\Psi^{(\lambda)}\left(\Phi^{(\lambda)}\right.$ and $\left.\Gamma^{(\lambda)}\right)$ faster than the estimator $\hat{\Psi}^{(\lambda)}\left(\hat{\Phi}^{(\lambda)}\right.$ and $\left.\hat{\Gamma}^{(\lambda)}\right)$ as the sample size $n$ becomes larger, when $\lambda \geq 1$.

When $\lambda<1$, we can calculate the refined estimators $\hat{\Psi}^{*(\lambda)}, \hat{\Phi}^{*(\lambda)}$ and $\hat{\Gamma}^{*(\lambda)}$ only the case of $\left\{\hat{p}_{i} \neq 0, \hat{p}_{. i} \neq 0\right\},\left\{\hat{p}_{i .}^{c} \neq 0, \hat{p}_{. i}^{c} \neq 0\right\}$ and $\left\{\hat{G}_{1(i)} \neq 0, \hat{G}_{2(i)} \neq 0\right\}$, respectively. On the other hand, the former estimators $\hat{\Psi}^{(\lambda)}, \hat{\Phi}^{(\lambda)}$ and $\hat{\Gamma}^{(\lambda)}$ can be calculated for the case of $\left\{\hat{p}_{i .}+\hat{p}_{. i} \neq 0\right\},\left\{\hat{p}_{i .}^{c}+\hat{p}_{. i}^{c} \neq 0\right\}$ and $\left\{\hat{G}_{1(i)}+\hat{G}_{2(i)} \neq 0\right\}$, respectively. This means that the calculable conditions of the refined estimators are different from that of the former estimators. Thus, it seems difficult to evaluate whether the improved estimators tend to approach to the true value faster than the former estimators by simulation study when $\lambda<1$.

Therefore, we recommend that the proposed estimators should be used for the case of $\lambda \geq 1$. Then these estimators work very well.

\section{Acknowledgments}

The authors would like to thank two anonymous referees for helpful comments.

\section{References}

[1] A. Agresti, Categorical Data Analysis, 3rd edition, Wiley, Hoboken, New Jersey (2013). 
[2] Y.M.M. Bishop, S.E. Fienberg and P.W. Holland, Discrete Multivariate Analysis: Theory and Practice, The MIT Press, Cambridge, Massachusetts (1975).

[3] T.R.C. Read and N. Cressie, Goodness-of-Fit Statistics for Discrete Multivariate Data, Springer, New York (1988).

[4] A. Stuart, A test for homogeneity of the marginal distributions in a twoway classification, Biometrika, 42 (1955), 412-416.

[5] K. Tahata, R. Tomisato and S. Tomizawa, An improved approximate unbiased estimator of log-odds ratio for $2 \times 2$ contingency tables, Advances and Applications in Statistics, 9 (2008), 1-12.

[6] S. Tomizawa, Measures of departure from marginal homogeneity for contingency tables with nominal categories, Journal of the Royal Statistical Society, Series D; The Statistician, 44 (1995), 425-439.

[7] S. Tomizawa and T. Makii, Generalized measures of departure from marginal homogeneity for contingency tables with nominal categories, Journal of Statistical Research, 35 (2001), 1-24.

[8] S. Tomizawa, N. Miyamoto and N. Ashihara, Measure of departure from marginal homogeneity for square contingency tables having ordered categories, Behaviormetrika, 30 (2003), 173-193.

[9] S. Tomizawa, N. Miyamoto and N. Ohba, Improved approximate unbiased estimators of measures of asymmetry for square contingency tables, Advances and Applications in Statistics, 7 (2007), 47-63. 
